\title{
Novel oral anticoagulation agents: New drugs create new paradigms
}

\author{
Jerrold H. Levy, MD, FAHA, FCCM
}

See related article on pages 1794-801.

In recent years managing patients' cardiovascular disease has increasingly focused on the critical role of preventing thrombus formation by pharmacologically inhibiting coagulation factors and platelets. From a perioperative perspective this is important because patients undergoing cardiac surgery often receive multiple antithrombotic and antiplatelet agents preoperatively that pose important considerations for management. ${ }^{1}$ Recently, several novel oral anticoagulation agents (NOACs) have been approved, including dabigatran, a direct thrombin inhibitor, and rivaroxaban, apixaban, and edoxaban, direct Factor Xa inhibitors that have multiple indications for either stroke prevention with nonvalvular atrial fibrillation or thromboprophylaxis. ${ }^{2,3}$ In this month's issue of the Journal, Kaneko ${ }^{4}$ reviews the perioperative considerations of NOACs. Important considerations and perspectives are as follows.

Unlike warfarin, NOACs have rapid and predictable onsets, and an offset more rapid than warfarin. Although monitoring is not required, all of these agents can be evaluated if needed, as Kaneko ${ }^{4}$ describes. For dabigatran, partial thromboplastin time can be used but thrombin times or a specific assay called Hemoclot assay (Aniara, West Chester, Ohio) should be considered for elective surgery. Rivaroxaban, apixiban, and other $\mathrm{Xa}$ inhibitors require specific calibrated antifactor-Xa assays similar to low-molecularweight heparin (LMWH) monitoring. Although concerns exist regarding the NOACs, managing the condition of any patient undergoing anticoagulant treatment in a perioperative setting can be challenging, because all anticoagulation agents can cause bleeding, and anticoagulation is for a specific prothrombotic issue. With any anticoagulant there are important considerations when they require surgery.

Despite growing concerns about NOACs, warfarin management still perplexes many clinicians because it is not completely reversible with standard therapies. Despite the continued use of fresh frozen plasma, 4-component

From the Duke University School of Medicine, Durham, NC.

Disclosures: J.H.L. serves on steering committees for Boehringer-Ingelheim, CSL Behring, J\&J, Grifols, and Portola.

Received for publication Sept 5, 2014; accepted for publication Sept 7, 2014

Address for reprints: Jerrold H. Levy, MD, FAHA, FCCM, Duke University Medical Center, 2301 Erwin Rd, 5691H HAFS, Box 3094, Durham, NC 27710 (E-mail: jerrold.levy@duke.edu).

J Thorac Cardiovasc Surg 2014;148:1802-3

$0022-5223 / \$ 36.00$

Copyright (c) 2014 by The American Association for Thoracic Surgery

http://dx.doi.org/10.1016/j.jtcvs.2014.09.003 prothrombin complex concentrates (PCCs) are recommended in recent guidelines, and have only recently become available in the United States. ${ }^{5,6}$ Unlike warfarin, NOACs have a direct effect on specific anticoagulation factors, and their effects normalize in $\sim 24$ to 48 hours after discontinuing the drug with normal renal function.

The major concern for clinicians is for emergency procedures or major bleeding. Importantly, there is perceived concern about the lack of a suitable antidote for these agents. However, LMWH, a ubiquitous anticoagulant in hospital settings, does not have an antidote and can cause major bleeding. However, there is increasing information available to facilitate managing patients receiving NOACs. ${ }^{3}$

Important management of NOACs includes discontinuing use, but if reversal is required, specific therapeutic approaches should be considered, such as the application of PCCs for reversal and/or bleeding. PCCs in the United States are available as 4-component or 3-component products. The 3-component PCCs are deficient in factor VII. We have reported the ability of PCCs to reverse rivaroxaban anticoagulation and there are increasing reports on their off-label use in bleeding cardiac surgical patients. $^{7-9}$

There are other important considerations when managing patients receiving NOACs. When patients bleed, there is variable correlation between laboratory tests and clinical bleeding. ${ }^{10}$ For example, in patients who have a lupus anticoagulant, coagulation tests are prolonged but in fact these patients are potentially hypercoagulable. Further, coagulation tests can be corrected without correcting the underlying hemostatic defect; we have reported data where recombinant Factor VIIa corrects the international normalized ratio of warfarin but only PCCs restore thrombin generation. ${ }^{11}$ The lack of correlation between in vivo bleeding and coagulation assay response was also observed in a recent trial comparing 4-factor PCC with plasma in patients treated with vitamin $\mathrm{K}$ antagonists with major bleeding. $^{12}$

What are the important perspectives for cardiac surgeons? It is highly unlikely that NOACs will ever replace warfarin for prosthetic valve or device anticoagulation because of the extensive experience using warfarin in this setting and the multiple targets of warfarin anticoagulation, including Factors II (prothrombin), VII, IX, and X. When managing patients taking NOACs who are bleeding or undergoing emergency surgery, a multimodal therapeutic plan should be considered and established a priori. ${ }^{3,13}$ In patients treated with dabigatran, hemodialysis may be a potential option that could be performed during 
cardiopulmonary bypass. ${ }^{14}$ The off-label use of PCCs should be considered when treating bleeding when it occurs in patients treated with NOACs and includes normalization of hemostasis with factor concentrates and transfusion factors. $^{3}$

Despite concerns about NOACs and bleeding, most studies suggest patients fare better on NOACs compared with warfarin. ${ }^{15}$ In 1 study of 27,419 patients treated up to 3 years, 1034 patients had 1121 major bleeds. The 30 -day mortality after the first major bleed was $9.1 \%$ in the dabigatran group compared with $13.0 \%$ in the warfarin group, and patients treated with dabigatran required a shorter intensive care unit stay compared with those treated with warfarin. ${ }^{15}$ Despite the relative safety of NOACs compared with warfarin, additional clinical studies are needed and are underway to determine optimal therapeutic approaches for bleeding when it occurs. For dabigatran, the Study of the Reversal Effects of Idarucizumab on Active Dabigatran is underway. ${ }^{16,17}$ Another novel agent for Xa inhibitor reversal that will also reverse LMWH is Andexanet alpha, which is also in clinical trials for rivaroxaban and apixiban reversal. ${ }^{18}$

\section{References}

1. Levy JH. Pharmacology and safety of new oral anticoagulants: the challenge of bleeding persists. Clin Lab Med. 2014;34:443-52.

2. Levy JH, Key NS, Azran MS. Novel oral anticoagulants: implications in the perioperative setting. Anesthesiology. 2010;113:726-45.

3. Levy JH, Faraoni D, Spring JL, Douketis JD, Samama CM. Managing new oral anticoagulants in the perioperative and intensive care unit setting. Anesthesiology. 2013:118:1466-74.

4. Kaneko T, Yammine M, Aranki SF. New oral anticoagulants-what the cardiothoracic surgeon needs to know. J Thorac Cardiovasc Surg. 2014;148:1794-801.

5. Douketis JD, Spyropoulos AC, Spencer FA, Mayr M, Jaffer AK, Eckman MH, et al. Perioperative management of antithrombotic therapy: antithrombotic therapy and prevention of thrombosis, 9th ed: American College of Chest
Physicians Evidence-Based Clinical Practice Guidelines. Chest. 2012;141: e326S-50S.

6. Levy JH, Tanaka KA, Dietrich W. Perioperative hemostatic management of patients treated with vitamin K antagonists. Anesthesiology. 2008;109:918-26.

7. Levi M, Moore KT, Castillejos CF, Kubitza D, Berkowitz SD, Goldhaber SZ, et al. Comparison of three-factor and four-factor prothrombin complex concentrates regarding reversal of the anticoagulant effects of rivaroxaban in healthy volunteers. J Thromb Haemost. 2014;12:1428-36.

8. Levy JH, Levi M. New oral anticoagulant-induced bleeding: clinical presentation and management. Clin Lab Med. 2014:34:575-86.

9. Song HK, Tibayan FA, Kahl EA, Sera VA, Slater MS, Deloughery TG, et al. Safety and efficacy of prothrombin complex concentrates for the treatment of coagulopathy after cardiac surgery. J Thorac Cardiovasc Surg. 2014;147: 1036-40.

10. Levy JH, Szlam F, Wolberg AS, Winkler A. Clinical use of the activated partial thromboplastin time and prothrombin time for screening: a review of the literature and current guidelines for testing. Clin Lab Med. 2014;34: 453-77.

11. Tanaka KA, Szlam F, Dickneite G, Levy JH. Effects of prothrombin complex concentrate and recombinant activated factor VII on vitamin $\mathrm{K}$ antagonist induced anticoagulation. Thromb Res. 2008;122:117-23.

12. Sarode R, Milling TJ Jr, Refaai MA, Mangione A, Schneider A, Durn BL, et al Efficacy and safety of a 4-factor prothrombin complex concentrate in patients on vitamin $\mathrm{K}$ antagonists presenting with major bleeding: a randomized, plasmacontrolled, Phase IIIb study. Circulation. 2013;128:1234-43.

13. Sniecinski RM, Levy JH. Bleeding and management of coagulopathy. J Thorac Cardiovasc Surg. 2011;142:662-7.

14. Khadzhynov D, Wagner F, Formella S, Wiegert E, Moschetti V, Slowinski T, et al Effective elimination of dabigatran by haemodialysis. A phase I single-centre study in patients with end-stage renal disease. Thromb Haemost. 2013;109: 596-605.

15. Majeed A, Hwang HG, Connolly SJ, Eikelboom JW, Ezekowitz MD, Wallentin L, et al. Management and outcomes of major bleeding during treatment with dabigatran or warfarin. Circulation. 2013;128:2325-32.

16. Schiele F, van Ryn J, Canada K, Newsome C, Sepulveda E, Park J, et al. A specific antidote for dabigatran: functional and structural characterization. Blood. 2013;121:3554-62

17. ClinicalTrials.gov. Reversal of dabigatran anticoagulant effect with idarucizumab. Available at: http://clinicaltrials.gov/ct2/show/NCT02104947? term=dabigatran+bleeding\&rank=3. Accessed September 26, 2014.

18. ClinicalTrials.gov. A study in older subject to evaluate the safety and ability of Andexanet alfa to reverse the anticoagulation effect of rivaroxaban. Available at: http://clinicaltrials.gov/ct2/show/NCT02220725?term=andexanet\&rank=1. Accessed September 26, 2014. 